\title{
Roles of ventral versus dorsal pathways in language production: an awake language mapping study
}

\author{
S.K. Ries ${ }^{1,2,3,{ }^{*}, \text { V. Piai }}{ }^{4,5}$, D. Perry ${ }^{6}$, S. Griffin ${ }^{7}$, K. Jordan ${ }^{8,9}$, R. Henry ${ }^{8}$, R.T. Knight ${ }^{7}$, and M. \\ Berger 6
}

1.School of Speech, Language, and Hearing Sciences, San Diego State University, United States

2.Center for Clinical and Cognitive Neuroscience, San Diego State University, United States ${ }^{3}$.Joint Doctoral Program in Language and Communicative Disorders, San Diego State University and University of California San Diego, United States ${ }^{4}$ Radboud University, Donders Institute for Brain Cognition and Behaviour, Donders Centre for Cognition, Nijmegen, the Netherlands ${ }^{5}$ Radboud university medical center, Donders Institute for Brain Cognition and Behaviour, Department of Medical Psychology, Nijmegen, the Netherlands ${ }^{6}$.University of California San Francisco, Department of Neurological Surgery, United States ${ }^{7}$ University of California Berkeley, Department of Psychology and the Helen Wills Neuroscience Institute, United States ${ }^{8}$.University of California San Francisco, Department of Neurology, United States ${ }^{9}$.Joint Doctoral Program in Bioengineering, University of California San Francisco and Berkeley, United States

\section{Abstract}

Human language is organized along two main processing streams connecting posterior temporal cortex and inferior frontal cortex in the left hemisphere, travelling dorsal and ventral to the Sylvian fissure. Some views propose a dorsal motor versus ventral semantic division. Others propose division by combinatorial mechanism, with the dorsal stream responsible for combining elements into a sequence and the ventral stream for forming semantic dependencies independent of sequential order. We acquired data from direct cortical stimulation in the left hemisphere in 17 neurosurgical patients and subcortical resection in a subset of 10 patients as part of awake language mapping. Two language tasks were employed: a sentence generation (SG) task tested the ability to form sequential and semantic dependencies, and a picture-word interference (PWI) task manipulated semantic interference. Results show increased error rates in the SG versus PWI task during subcortical testing in the dorsal stream territory, and high error rates in both tasks in the ventral stream territory. Connectivity maps derived from diffusion imaging and seeded in the tumor sites show that patients with more errors in the SG than in the PWI task had tumor locations associated with a dorsal stream connectivity pattern. Patients with the opposite pattern of results had tumor locations associated with a more ventral stream connectivity pattern. These findings provide initial evidence using fiber tract disruption with electrical stimulation that the dorsal

*Corresponding author: Stephanie K. Ries, PhD, Assistant Professor, School of Speech, Language, and Hearing Sciences, San Diego State University, SLHS room \#239, 5500 Campanile Drive, San Diego, CA 92182-1518.

Publisher's Disclaimer: This is a PDF file of an unedited manuscript that has been accepted for publication. As a service to our customers we are providing this early version of the manuscript. The manuscript will undergo copyediting, typesetting, and review of the resulting proof before it is published in its final citable form. Please note that during the production process errors may be discovered which could affect the content, and all legal disclaimers that apply to the journal pertain. 
pathways are critical for organizing words in a sequence necessary for sentence generation, and the ventral pathways are critical for processing semantic dependencies.

\section{Introduction}

The neural basis for language is thought to be organized along two main processing streams connecting the posterior temporal cortex to the inferior frontal cortex in the left hemisphere: one travelling dorsally and the other ventrally, relative to the Sylvian fissure (Bornkessel-

Schlesewsky et al., 2015; Fridriksson et al., 2018, 2016; Hickok \& Poeppel, 2007;

Rauschecker \& Scott, 2009; Saur et al., 2008; Ueno, Saito, Rogers, \& Lambon Ralph, 2011).

Most studies addressing this dorsal/ventral division have investigated speech perception processes, rarely taking speech production into account (although see, Fridriksson et al., 2018, 2016; Hickok, 2012; Roelofs, 2014; Ueno et al., 2011). Furthermore, the causal roles of the ventral and dorsal language pathways have seldom been tested through direct brain stimulation (Duffau et al., 2002; Duffau, Moritz-Gasser, \& Mandonnet, 2014). We report initial steps to address the causal roles of ventral and dorsal pathways in language production through direct cortical electrical stimulation (DCES) and subcortical stimulation and resection as part of awake language mapping during surgery for brain tumor.

Carl Wernicke and Ludwig Lichtheim in the $19^{\text {th }}$ century proposed two pathways linking "auditory word images" stored in Wernicke's area to "motor word images" stored in Broca's area. In this dual-pathway model based on lesion studies, "auditory word images" were converted into "motor word images" through a phonological pathway (later defined as the arcuate fasciculus) or indirectly through a semantic pathway involving a distributed conceptual center or network (Lichtheim, 1885). This dissociation was based mainly on the pattern of errors observed in conduction aphasia, wherein relatively fluent, although paraphasic, spontaneous speech and preserved auditory comprehension can be dissociated from impaired speech repetition (Tippett \& Hillis, 2016). These early neurolinguistic models lacked anatomical specification (Chang, Raygor, \& Berger, 2015). Contemporary models include results from neuroimaging studies and are inspired from the dorsal (where) / ventral (what) division of the visual (Ungerleider \& Mishkin, 1982) and auditory (Rauschecker \& Tian, 2000) systems in the brain. Hickok and Poeppel (2007) proposed a neurobiological model following a motor (dorsal) versus conceptual/semantic (ventral) division. The dorsal pathway includes the arcuate and superior longitudinal fasciculi, and the posterior superior temporal lobe (including area Spt at the temporoparietal junction in the Sylvian fissure) terminating in the inferior frontal gyrus (IFG) and premotor cortex, as shown by a neuroimaging study combining functional magnetic resonance imaging with diffusion imaging results (Saur et al., 2008). The ventral pathway involves the middle and inferior longitudinal fasciculi but also the uncinate fasciculus (Fridriksson et al., 2016) and inferior fronto-occipital fasciculus (Martino, Brogna, Robles, Vergani, \& Duffau, 2010), as well as the superior temporal gyrus ending near anterior portions of the inferior frontal gyrus (Broca's area: pars orbitalis and triangularis) (Saur et al., 2008). The dorsal pathway is linked to sensori-motor mapping of sound to articulation (Saur et al., 2008), and auditory feedback control in speech production (Hickok, 2012), making it important for supporting repetition (Ueno et al., 2011). By contrast, the ventral stream has been associated with the 
ability to map auditory input onto conceptual and semantic representations, as well as syntactic processing (Hickok \& Poeppel, 2004, 2007; Ueno et al., 2011). We note that some researchers argue that a secondary dorsal stream pathway supports the mapping of lexicalsemantic representations onto speech output processes (Glasser \& Rilling, 2008; Roelofs, 2014).

Other neuroimaging studies have proposed that the dorsal stream also plays a role in syntactic processing (Friederici, Bahlmann, Heim, Schubotz, \& Anwander, 2006; Friederici, Makuuchi, \& Bahlmann, 2009; Bornkessel-Schlesewsky et al., 2015; Friederici, 2012), although some models suggest a dissociation between complex and simple syntactic processing with the dorsal stream being primarily involved in complex syntax (Friederici et al., 2006, 2012). Interestingly, damage to the dorsal but not ventral pathway correlates with deficits in syntactic processing in primary progressive aphasia (Wilson et al., 2011). Inspired by auditory processing research in non-human primates (Rauschecker \& Tian, 2000), Bornkessel-Schlesewsky et al. (2015) proposed a dorsal/ventral division of auditory language processing (see also, Rauschecker \& Scott, 2009). According to this proposal, the dorsal stream contributes to combining elements into a sequence (i.e., phonemes but also sentences), and the ventral stream contributes to the formation of dependencies independent of sequential order (i.e., semantics).

Although dual stream models have been mainly focused on language perception, recent dual-stream models of language production have been proposed (Fridriksson et al., 2016; Hickok, 2012; Roelofs, 2014; Ueno et al., 2011). These models generally propose that speech repetition and auditory feedback control are enabled through the dorsal stream (Hickok, 2012; Ueno et al., 2011). In addition, these models generally argue that conceptualto-lexical mapping in language production is implemented along the ventral stream similarly as lexical-to-conceptual mapping in speech perception (Fridriksson et al., 2016; Hickok, 2012; Roelofs, 2014; Ueno et al., 2011), even if (Roelofs, 2014) proposes the connection to speech output is subserved by a secondary dorsal stream. . However, whether sequential word production in a sentence would involve the dorsal stream, the ventral stream, or both is unclear. Existing dual-stream models offer diverging views with respect to the neural substrate needed for syntactic comprehension, with some arguing for a role of the dorsal stream (Bornkessel-Schlesewsky et al., 2015), and others arguing that the ventral stream is primarily responsible (Fridriksson et al., 2016; Hickok \& Poeppel, 2004; Saur et al., 2008).

This study tests for a dorsal/ventral division in language production and for a causal role of the dorsal pathway in word sequence formation by testing language production abilities during DCES (Penfield \& Roberts, 1959) and subcortical resection and stimulation in patients undergoing surgery for brain tumor removal. This technique is typically used to preserve eloquent cortex (i.e., cortical regions supporting language, motor, and sensory functions) in patients undergoing resective surgery for brain tumor removal or removal of the epileptogenic zone in refractory epilepsy (see Methods).

Awake language mapping has recently included language testing during subcortical stimulation, in addition to standard cortical stimulation (Hugues Duffau et al., 2002; Hugues Duffau, Gatignol, Denvil, Lopes, \& Capelle, 2003; Hugues Duffau et al., 2005; Mandonnet, 
Nouet, Gatignol, Capelle, \& Duffau, 2007). This approach is used to preserve critical white matter pathways during resection (see also Caverzasi et al., 2016), and have led to the proposal of a hodotopical ${ }^{1}$ map combining cortical regions and white matter pathways supporting language (Duffau, 2015; Duffau, Moritz-Gasser, \& Mandonnet, 2014, see also Catani \& Mesulam, 2008; Turken \& Dronkers, 2011).

We use two tasks: 1) picture-word interference (PWI), manipulating meaning dependencies (Glaser \& Düngelhoff, 1984; Lupker, 1979) and 2) simple sentence generation, requiring ordering words in a sequence.

In the PWI task, used extensively in the field of psycholinguistics, pictures are presented with superimposed distractor words (e.g., Bürki, 2017; Costa, Alario, \& Caramazza, 2005; Piai, Roelofs, Acheson, \& Takashima, 2013; Piai, Roelofs, Jensen, Schoffelen, \& Bonnefond, 2014; Piai, Roelofs, \& Schriefers, 2015; Roelofs \& Piai, 2015, 2017). In the semantic version of the task (used here), the distractor words can be semantically related to the picture (e.g., picture of a dog, distractor word: "cat") or unrelated (e.g., picture of a dog, distractor word: "chair"). Naming the picture takes longer and error rates are higher in the semantically-related, compared to the unrelated condition. This is interpreted as increased competition for word selection caused by over-activation of semantically-related alternatives in the semantically-related compared to the unrelated condition. The experimental manipulation employs meaning dependencies, and therefore should involve ventral white matter pathways. In support of this hypothesis, this task has been shown to elicit differential hemodynamic responses in several brain regions, including left temporal regions (anterior and posterior superior temporal gyrus, middle temporal gyrus), and frontal regions (orbitomedial prefrontal cortex) (Piai et al., 2013; Zubicaray, Wilson, McMahon, \& Muthiah, 2001). We note the medial frontal cortex, and in particular the anterior cingulate cortex has been associated with control processes engaged in this paradigm (Piai et al., 2013). In addition, lesions in the left temporal lobe have been shown to be associated with larger semantic interference effects (Piai \& Knight, 2017).

In the second task, patients were asked to produce simple sentences, containing a subject, verb, and object when needed (e.g., "the woman is writing a letter"), in response to action pictures. This task involves both access to meaning and ordering elements in a sequence. Accordingly, we hypothesized that overt sentence generation would engage both the ventral and dorsal streams. In support of the engagement of the dorsal stream in this task, overt sentence generation has been shown to elicit stronger fMRI responses than sentence reading in the left posterior IFG (BA 44/45), medial frontal cortex (BA 6), and superior parietal lobule (BA7) (Haller, Radue, Erb, Grodd, \& Kircher, 2005; see also Indefrey et al., 2001, for a positron emission tomography study supporting the role of left BA 44 and BA 6 in syntactic encoding during production). In addition and as noted above, damage to the superior longitudinal fasciculus as measured through diffusion tensor imaging has been associated with deficits in production and comprehension of syntax in patients with primary progressive aphasia (Wilson et al., 2011). However, cortical regions along the ventral stream

${ }^{1}$ A hodotopical map combines the pathway (from the Greek hodos $=$ road or path) and the area (from the Greek topos = place) involved in a given function (Duffau, Moritz-Gasser, \& Mandonnet, 2014). 
such as the left middle temporal gyrus (BA 21) (Segaert, Menenti, Weber, Petersson, \& Hagoort, 2012) and the left temporal pole (Pylkkänen, Bemis, \& Blanco Elorrieta, 2014) were also associated with sentence or phrase production, suggesting the ventral stream may also play a role in sentence generation. We hypothesized that the sentence generation task would be dependent on both the dorsal and ventral streams, but that the PWI task would involve the ventral stream more strongly than the dorsal stream.

\section{Materials and Methods}

\subsection{Participants}

Seventeen patients undergoing resective surgery for tumor removal in the left hemisphere participated (11 males; mean [sd] age at testing: 40 [14] years, and education: 16 [2] years) ${ }^{2}$. All patients were fluent in English to the level of native proficiency (one patient was not a native English speaker but was proficient in English). Of the 17 patients, 16 were stimulated cortically and 10 were tested during subcortical resection in one or both tasks. Etiologies, tumor grade, and resected tumor general locations are indicated in Table 1. Overlays of tumor sites are also presented in Figure 1. Of the 10 patients tested during subcortical resection, 5 had tumors above the Sylvian fissure and were classified as "dorsal", and 5 had tumors below the Sylvian fissure and were classified as "ventral" (see Figure 2 for tumor site overlays for the dorsal and ventral groups). The study was approved by the UCSF and UC Berkeley Institutional Review Boards, and all participants gave written informed consent. Analysis of de-identified data took place at San Diego State University, UC Berkeley, and UCSF.

\subsection{Surgical procedures}

All patients underwent craniotomy using monitored anesthesia care: local anesthetic infiltration was applied to the scalp. Patients were sedated with either Propofol or dexmedetomidine at the start of the procedure. Surgical exposure was tailored to the target lesion. Intraoperative language mapping with DCES was performed in all but one patient, who had a seizure during motor mapping and did not participate in further testing. Patients were fully awake for the DCES mapping; intraoperative electrocorticography was used to monitor for stimulation-induced after-discharges. During this procedure patients perform various tasks testing for motor, sensory, and basic language functions (counting, reading, and picture naming) while bipolar electrical stimulation $(60 \mathrm{~Hz})$ is applied for two second intervals to their exposed cortical surface using a manual handheld probe at a tailored intensity (typically $\sim 3-4 \mathrm{~mA}$ ). Epileptic after-discharges are monitored during the procedure using electrocorticography. Positive sites (i.e., sites where stimulation causes a disruption of the tested function) are marked using small tickets intraoperatively. Clinical intraoperative language tasks included counting, picture naming (line-drawings from Snodgrass \& Vanderwart, 1980), and reading. These tasks were used both during cortical stimulation mapping (before resection) to identify cortical sites critical for receptive and expressive language, and during subcortical resection to identify white matter pathways involved in

\footnotetext{
${ }^{2}$ We also tested 2 patients with right hemisphere lesions (Pt 3 and 14) who were not included in the analyses. See supplementary materials (Figure S1).
} 
these processes. Essential language sites were defined as those resulting in a loss of function in at least 2 of 3 stimulations. Every effort was made to avoid or limit the resection of these essential language sites while maximizing tumor removal. In addition, the patients participated in one or both of our experimental tasks during cortical and/or subcortical testing. After mapping, patients were re-sedated with either propofol or dexmedetomidine for the remainder of the procedure. Resection was performed with an ultrasonic aspirator guided by intraoperative neuro-navigation.

\subsection{Tasks}

We used a picture-word interference (PWI) task manipulating semantic interference and a sentence generation task testing the ability to form sequential dependencies as well as meaning dependencies. Both tasks were practiced by each patient within two days prior to surgery to enhance intra-operative performance.

For the PWI task, the stimuli were 20 colored photographs on a white background issued from the BOSS database (Brodeur, Dionne-Dostie, Montreuil, \& Lepage, 2010) with distractor words superimposed. The pictures belonged to 5 different semantic categories with 4 items per category (see Supplementary Materials). Distractor words were the picture names from either semantically related pictures (related condition; e.g., picture of a banana, "pear" superimposed), or semantically and phonologically unrelated pictures in the set (unrelated condition; e.g., picture of a banana, "arm" superimposed). All distractor words belonged to the response set; each list contained all pictures and picture names, once in each condition. Stimuli were presented in the center of the screen on a gray background; a white distractor word was centered on each picture. Trials were randomized using Mix (van Casteren \& Davis, 2006) to create 5 different lists. Each patient only saw one list during the intraoperative testing; lists were counter-balanced across patients. Since the PWI task was practiced before surgery, the same stimuli were seen during the familiarization and the intraoperative testing but there were 40 additional items in the familiarization phase. The 60 pictures belonged to 10 semantic categories with 6 pictures per category. The pictures were also presented one additional time in the familiarization testing, as there was a neutral condition in which the distractor was a string of X's.

For the sentence generation task, the stimuli were 32 line drawings issued from a published and normed database (Masterson \& Druks, 1998), representing easily identifiable actions (e.g., a man going down the stairs). Half of the pictures represented intransitive action verbs (e.g., swimming), and half represented transitive action verbs (e.g., lighting a candle).

Because the stimuli had been normed for isolated verb production only, we did a control-test in which we asked 10 control participants (mean [sd] age: 25[5] years; 3 males) to produce complete simple sentences for 125 action pictures issued from the database (Masterson \& Druks, 1998). As experimental items, we selected 32 pictures with minimum $80 \%$ overlap between participants in terms of subject (e.g., for the gender choice, "she" being considered equivalent to "the woman"), verb stem (e.g., "shaved" and "is shaving" being considered equivalent), and object choices for transitive verbs. The average lemma frequency (obtained from the SUBTL database, Brysbaert \& New, 2009) tended to be higher for the verbs elicited in the sentence generation task (mean lemma frequency per million=131.36, $\mathrm{SD}=$ 
128.82) than for the nouns elicited in the PWI task (intraoperative list: mean [sd] lemma frequency per million $=73.99$ [114.93], $\mathrm{t}(43.95)=-1.671, \mathrm{p}=.102$; familiarization list: mean[sd] lemma frequency per million $=59.54$ [106.21], $\mathrm{t}(53.83)=2.702, \mathrm{p}=.009)$. Stimuli were presented at the center of the screen on a gray background. The trials were pseudorandomized using Mix (van Casteren \& Davis, 2006) such that no more than 2 transitive or intransitive action-representing picture were presented in a row. Ten different lists were created, counter-balanced across participants and used for both the familiarization and intraoperative testing (different lists were used within-participant).

\subsection{Procedure}

Stimulus presentation was controlled by Psyscope (Cohen, MacWhinney, Flatt, \& Provost, 1993) during intraoperative testing, and by E-Prime 2.0 Professional software (Psychology Software Tools, Inc., Pittsburgh,PA) during familiarization testing. Vocal responses were recorded through a high-definition microphone (Zoom H2n, ZOOM North America, Hauppauge, NY; sampling rate: $48 \mathrm{kHz}$ ) placed close to the stimulus presentation computer during the intraoperative procedure, and directly through E-Prime (sampling rate: 22,050 $\mathrm{Hz}$ ) during the familiarization testing. During intraoperative testing, a wireless microphone placed close to the patient's mouth on the bed amplified their voice to ensure communication with the neurosurgeon, enhancing the clarity of sound recording. During intraoperative testing, trials were as follows: a blank screen was presented for $6000 \mathrm{msec}$. The sound of a slide projector changing ( $1.5 \mathrm{sec}$. duration) announced the upcoming stimulus (volume was optimized for each patient). Then the stimulus was presented for a duration of at least $4000 \mathrm{msec}$ (stimulation duration could be lengthened depending on the patient). Stimuli were presented sideways on a computer screen oriented toward the patient at a comfortable viewing distance. The patient laid sideways on the operating room bed with his/her head oriented with the surgical exposure facing upward the neurosurgeon. The neuropsychologist, present during the entire language testing session, ensured that the patient could clearly see the stimuli before the experiment started.

During the familiarization testing in the PWI task, trials were as follows: there was a variable inter-trial interval which lasted between 1.7 and $2.1 \mathrm{sec}$. during which a centrally positioned crosshair (fixation cross) was presented. Then, the stimulus was presented for 2 seconds.

During familiarization testing in the sentence generation task, trials were as follows: a centrally positioned crosshair was presented for $900 \mathrm{msec}$., followed by the stimulus for $3000 \mathrm{msec}$., followed by a white screen for $3000 \mathrm{msec}$. Then, the next trial began automatically. The patient was comfortably seated in a private room and the stimuli were presented on a computer screen at a comfortable viewing distance in front of the patient.

In the PWI task, the instructions were to name the picture while ignoring the distractor words. In the sentence generation task, the instructions were to produce a simple but complete sentence describing the picture. For example, when seeing a picture of a man sleeping, the patient could say "the man is sleeping" or "a man is sleeping". The experimenter emphasized speed and accuracy for both tasks. 


\subsection{Neuroimaging, lesion mapping, and diffusion processing.}

All patients underwent Magnetic Resonance Imaging (MRI) on a 3T General Electric Medical Systems scanner (Discovery MR750) within the week preceding surgery. Datasets acquired included: a High Angular Resolution Diffusion Imaging (HARDI) sequence (TR/TE $=6425 / 80 \mathrm{~ms}, 50$ axial slices, $2.2 \mathrm{~mm}$ inplane resolution (interpolated to $1.1 \mathrm{~mm}$ ), $2 \mathrm{~mm}$ slice thickness, $\mathrm{b}$-value $2000 \mathrm{~s} / \mathrm{mm}^{2}, 55$ diffusion gradients, 1 minimally diffusion weighted "B0" image), and T1, T2 and FLAIR sequences.

Lesion (i.e., tumor site) masks were manually delineated slice-by-slice using MRIcron (http://www.mccauslandcenter.sc.edu/mricro/mricron/) by a trained research assistant (S.G.) directed by a neurologist (R.T.K.) using input from T1, T2, and FLAIR images after normalization to MNI space (Montreal Neurological Institute).

HARDI datasets were corrected for motion and eddy current distortion using the FMRIB Software Library (FSL) (Jenkinson, Beckmann, Behrens, Woolrich, \& Smith, 2012) and the gradient table rotated (Leemans \& Jones, 2009). A tensor model was then fit to the preprocessed data using the open-source package Diffusion Imaging in Python (Dipy) (Garyfallidis et al., 2014) to generate diffusion metrics. All non-brain tissue was removed from diffusion-derived images using a brain mask generated from skull-stripping the B0 image (Smith, 2002). A diffeomorphic registration was performed between either the B0 image or the fractional anisotropy (FA) image and the MRI contrast on which the lesion reconstruction volume was drawn (FA for T1 images and B0 for T2 or FLAIR) using the Symmetric Diffeomorphic Registration in 3D (B. B. Avants, Epstein, Grossman, \& Gee, 2008; Brian B. Avants, Tustison, \& Song, 2009), implemented in Dipy (Garyfallidis et al., 2014). The lesion reconstruction volume was moved to diffusion space using the resulting transformation.

Whole-brain residual bootstrap probabilistic q-ball tractography (Berman et al., 2008) was performed by seeding all of the voxels with fractional anisotropy greater than 0.15 at a density of 1 seed per voxel with the tracking parameters described in (Caverzasi et al., 2016). The lesion reconstruction volume was targeted and outlier streamlines removed by setting a Cluster Confidence Index (default parameters: theta $=5, \mathrm{k}=1$ ) threshold (Jordan, Amirbekian, Keshavan, \& Henry, 2018) subjectively using the Trackvis viewer (Wang, Van Wedeen). Any streamlines less than $40 \mathrm{~mm}$ in length were excluded. Streamline datasets were converted to binary masks, transformed back to MNI space using the reverse diffeomorphic registration, and spatially summed to create density maps of lesion connectivity for the groups of interest (Figure 5, see supplementary materials for lesion connectivity for the whole patient group, Figure S2).

\subsection{Analysis}

Analysis were performed on response accuracy rates. Errors were determined using the vocal recordings and included paraphasias (semantic, phonological, remote, or neologistic), grammatical errors, hesitations, or no responses (including when the patient said he/she did not know the answer). We also distinguished verb vs. non-verb errors. Verb errors included the production of a wrong verb, phonological errors in the verb production, omission of the 
auxiliary verb, or failures to retrieve the verb (hesitations were excluded from this analysis of verb errors because it was often unclear what part of the sentence caused the hesitation). We could not analyze the effect of the parameters of interest (Task and Stream) on the different types of errors that were made because of the low number of observations, but report the overall distribution of error types per Task and per Stream in the supplementary materials.

Cortical stimulation sites were labelled by a neurosurgeon (M.B.) and classified as being on the dorsal or ventral stream following reviews and studies in the field (Hickok \& Poeppel, 2007; Saur et al., 2008; see Table 2). Error-rates per task per region are also presented in the supplementary materials (Table S4). For subcortical testing, the ventral/dorsal distinction was made based on tumor location as the patient was being tested during tumor resection. Tumor sites dorsal to the Sylvian fissure were classified as dorsal and tumor sites ventral to the Sylvian fissure were classified as ventral (Fig. 2).

Statistical analysis was performed with $\mathrm{R}$ version 3.3.2 (R Core Team, 2016), using packages "Ime4" for the mixed effect models (Bates, Mächler, Bolker, \& Walker, 2014) and "car" to compute analysis of deviance tables for the fixed effects of the mixed effect models (Fox \& Weisberg, 2011). We analyzed accuracy rates using logistic mixed effect models (Baayen, Davidson, \& Bates, 2008; Jaeger, 2008). Mixed effect models rely on single-trial data rather than on averages over participants or items, and are also free from the assumptions of homogenous variance and sphericity that are inherent to the more classic ANOVA (Pinheiro \& Bates, 2000). One major advantage for the current study is that these models allow for varying number of trials per condition for each participant and item and do not require for each participant or item to have values in each condition. We controlled for random effects of patient (including a random slope for Task) and items. We could not control for a random effect of items nor add by-patient random slopes for the effects of interest due to time constraints in the intraoperative testing leading insufficient statistical power. Thus, we report individual patient data in Figures 3 and 4.

In addition, we tested for an effect of the experimental manipulations in each task (i.e., relation between picture name and distractor in the PWI task and transitivity of the verb in the sentence generation task, hereafter referred to as "Condition") on accuracy rates for the preoperative data. We performed one logistic mixed effect model in each task testing for a fixed effect of Condition and controlled for random effects of patient (including a random slope for Condition when possible ${ }^{3}$ ) and items. During intraoperative testing, we did not have sufficient statistical power to analyze the effect of Condition within task and collapsed across conditions for analysis.

Finally, we could not separate trials during which electrical stimulation occurred from those during which only ultrasonic resection was performed during subcortical resection. Electrical stimulation was performed on only $20 \%$ of the 225 trials available for analysis overall, with some patients having zero electrical stimulation trials, so we collapsed

\footnotetext{
${ }^{3}$ There was an insufficient number of data points in one patient in the PWI during preoperative testing as this patient only performed 40 trials instead of 180 as the other patients, and was exposed to 2 out of the 3 conditions (related and unrelated, but not to the X string).
} 
electrical stimulation and resection trials for the subcortical analysis. We nevertheless report descriptive results including only the trials during which electrical stimulation was performed subcortically in the supplementary materials (section 3 ). These results show the same pattern of effects for each patient as reported below when collapsing the stimulation and resection trials. Electric stimulation is the gold standard procedure to briefly disrupt neuronal activity in a circumscribed brain area without altering the tissue, and is used prior to resection cortically, or before continuing resection in particular directions subcortically (for example, when approaching key white matter pathways such as the cortico-spinal motor tract delineated through preoperative diffusion imaging). However, as reported by Sierpowska et al. (2017), the ultrasonic generator used for the resection has been shown to cause effects in adjacent tissue similar to the stimulation induced by the Ojemann cortical electrical stimulator, used here (Carrabba et al., 2008). Even though the ultrasonic generator also transiently disrupts brain tissue and is typically not used as a clinical tool to test brain function, its causal disrupting effect is similar to the standard electric stimulation used in the current study. This permitted us to collapse both types of trials. We therefore refer to these results as the subcortical disruption results, and not to the subcortical stimulation results.

We compared accuracy rates between testing times: familiarization testing, intraoperative cortical testing, and intraoperative subcortical testing. P-values were obtained using type-III (because of the presence of an interaction) analyses of deviance tables providing Wald chisquare tests for the fixed effects. For all models, we report Wald chi-square values and associated p-values as well as raw beta estimates, $95 \%$ confidence intervals around these estimates, standard errors, Wald Z, and associated p-values.

\section{Results}

\subsection{Preoperative results}

The median error rates per task were low during preoperative testing (PWI: 5.00\% median error rate, inter-quartile interval, IQI $=[2.78 \%-10.56 \%]$; Sentence Generation: $6.25 \%$ median error rate, $\mathrm{IQI}=[3.13 \%-15.63 \%])$ and no significant effects were found.

There was no effect of Task on accuracy rates (Wald $\chi^{2}(1)=0.25, \mathrm{p}=.615$ ): patients did not make more errors in one task versus the other. There was also no effect of Stream (Wald $\chi^{2}$ $(1)=0.11, p=.720)$ : patients did not make more errors if their tumor was located above versus below the Sylvian fissure. Finally, there was no interaction between Task and Stream (Wald $\left.\chi^{2}(1)=0.12, p=.728\right)$.

Within-task analyses did not reveal any effect of Condition. There was no effect of verb transitivity in the sentence generation task (Wald $\chi^{2}(1)=1.77, \mathrm{p}=.183$ ), and no effect of picture-distractor relatedness ${ }^{4}$ in the PWI task (Wald $\chi^{2}(1)=1.46, p=.227$ ). We report the median error rates per condition in the PWI task and per Stream in the supplementary materials (Table S5).

\footnotetext{
${ }^{4}$ We excluded the neutral condition from this analysis because one patient (Pt10) practiced with the intraoperative version of the PWI task, which did not contain this third condition. Here the conditions compared are "related" vs. "unrelated".
} 


\subsection{Intraoperative results}

There was an effect of Testing Time on accuracy rates (Wald $\chi^{2}(2)=97.18, p<.001$ ). Accuracy rates were higher during preoperative testing than during cortical testing $\left(\beta_{\text {raw }}=\right.$ $0.672, \mathrm{CI}=[0.4320 .912], \mathrm{SD}=0.123$, Wald $\mathrm{Z}=5.490, \mathrm{P}<.001)$, and lower during subcortical testing compared to cortical testing $\left(\beta_{\text {raw }}=-1.092, \mathrm{CI}=[-1.511-0.673], \mathrm{SD}=\right.$ 0.214 , Wald $\mathrm{Z}=-5.110, \mathrm{P}<.001$ ). Thus, participants made more errors during subcortical testing than during cortical testing and during the awake language mapping procedure overall compared to the preoperative testing.

3.2.1. Cortical testing-During cortical stimulation, there was no effect of Task (Wald $\chi^{2}(1)=0.168, p=.682$ ), a main effect of Stream (Wald $\chi^{2}(1)=3.84, p=.050$, with more errors during ventral than dorsal stream stimulations), and a significant interaction between Task and Stream on accuracy rates (Wald $\chi^{2}(1)=6.52, p=.011$, see Fig. 3 for by-patient results). When we examined for an effect of Task on accuracy rates within dorsal and ventral stream stimulation, we found no effect of Task during dorsal stream stimulation (Wald $\chi^{2}$ $(1)=0.18, p_{c}>1$, Bonferroni-corrected $\mathrm{p}$-value ${ }^{5}$ for multiple comparisons), but found a significant effect of Task during ventral stream stimulation (Wald $\chi^{2}(1)=9.37, \mathrm{p}_{\mathrm{c}}=.004$, Bonferroni-corrected $\mathrm{p}$-value), where the patients were less accurate in the Sentence Generation task versus the PWI task during ventral stream stimulation $\left(\beta_{\text {raw }}=-1.191, \mathrm{CI}=\right.$ [-1.953 -0.428], $\mathrm{SD}=0.389$, Wald $\mathrm{Z}=-3.06, \mathrm{P}=.002$, see Table 3 and Fig. 3 ). We note however that the error rates were very low in the PWI task during cortical stimulation of brain regions along the dorsal and ventral streams (dorsal stream median error rate: $0 \%$, $\mathrm{IQI}=[0 \%-16.67 \%]$; ventral stream median error rate: $0 \%$, IQI= [0\%-18.25\%]). The distribution of the error types per Task and per Stream as well as the median error rates per stimulated brain region are presented in the supplementary materials (Tables S1 and S4).

3.2.2. Subcortical testing-During subcortical testing, there was a main effect of Task on accuracy rates (Wald $\chi^{2}(1)=7.410, p=0.006$ ): the sentence generation task elicited more errors than the PWI task (see Fig. 4). There was also a marginal effect of Stream (Wald $\chi^{2}$ $(1)=2.763, p=0.096$ ): performance tended to be lower in the territory of ventral stream pathways compared to dorsal stream pathways $\left(\beta_{\text {raw }}=-1.870, \mathrm{CI}=[-4.0750 .335], \mathrm{SD}=\right.$ 1.125 , Wald $\mathrm{Z}=-1.662, \mathrm{P}=.097)$. Critically, there was an interaction between Task and Stream (Wald $\chi^{2}(1)=8.624, \mathrm{p}=.003, \beta_{\text {raw }}=2.835, \mathrm{CI}=[0.9434 .727], \mathrm{SD}=0.965$, Wald $\mathrm{Z}=2.937, \mathrm{P}=.003$ ): There were more errors in the sentence generation task than in the PWI task in the territory of dorsal stream pathways (i.e., for tumors located above the Sylvian fissure). The opposite pattern was observed in the territory of ventral stream pathways (see Fig 4 and Table 4). When we examined for an effect of Task on accuracy rates within dorsal and ventral stream, we found an effect of Task in the dorsal stream (Wald $\chi^{2}(1)=7.47, \mathrm{p}_{\mathrm{c}}=$. 012, Bonferroni-corrected p-value), but found no significant effect of Task in the ventral stream (Wald $\chi^{2}(1)=1.42, \mathrm{p}_{\mathrm{c}}=.468$, Bonferroni-corrected $\mathrm{p}$-value). The distribution of the error types per Task and per Stream are presented in the supplementary materials.

\footnotetext{
${ }^{5} \mathrm{P}$-values were multiplied by 2 for the by-Stream comparisons.
} 


\subsection{Diffusion imaging data in relation to behavioral data}

The patients with both PWI and Sentence Generation subcortical testing $(\mathrm{N}=7)$ were split into a group that had a higher error rate in PWI than Sentence Generation $(\mathrm{N}=4)$ and a group that had a higher error rate in Sentence Generation than PWI $(\mathrm{N}=3)$ to evaluate differences between the tumor connectivity patterns of the groups. Importantly, for this analysis, groups were not separated based on tumor location but on behavioral profiles. As can be seen on the connectivity maps (Fig. 5), the patients with higher error rates in Sentence Generation versus PWI had tumor locations associated with a dorsal stream connectivity pattern (in green), meaning the streamlines intersecting tumor locations, as indicated by the tumor reconstructions, corresponded to dorsal pathways. This means that the pathways that were potentially stimulated in patients who had higher error rates in Sentence Generation compared to PWI were likely dorsal. By contrast, the patients with higher error rates in PWI versus Sentence Generation had tumor locations associated with a broader though more ventral stream connectivity pattern (in yellow). This means that the pathways that were potentially stimulated in patients who had higher error rates in PWI compared to Sentence Generation tended to be more ventral, compared to the pathways involved for the patients with the opposite pattern of results.

\section{Discussion}

Our results provide a rare window into the causal roles of the dorsal and ventral streams in language production through the lens of awake language mapping using cortical stimulation and subcortical ultrasound resection and stimulation. Notably, all subjects were tested presurgically and did not show any task effects nor any interaction with tumor location then, thus controlling for the effect of tumor per se. By contrast, the behavioral patterns of patients changed intraoperatively depending on the cortical and subcortical areas that were being tested and the task performed. Our results support the claim that dorsal stream pathways are engaged in organizing elements in a sequence, and that ventral stream pathways are engaged in the processing of meaning dependencies.

\section{Role of the ventral stream in language production}

There were more errors during ventral stream testing than during dorsal stream testing: a main effect of Stream was present during cortical testing and trended to significance during subcortical testing. These results support a role of the ventral stream cortical and subcortical pathways in both single word production and in sentence generation. Dual stream models of language perception have associated the ventral stream with lexical-to-semantic mapping (e.g., Hickok \& Poeppel, 2004, 2007; Rauschecker \& Scott, 2009; Saur et al., 2008) or the formation of meaning dependencies independent of sequential order (Bornkessel-

Schlesewsky et al., 2015). A stroke lesion study (Fridriksson et al., 2016) including multiple language production tasks argued that the ventral stream supports semantic-to-lexical mapping in language production, similarly to supporting lexical-to-semantic mapping in language perception. Our results are in agreement with this interpretation given the two language production tasks we used involved semantic-to-lexical mapping. We note that there are differences between dual stream production models as to which tract links lexicalsemantic representations to speech output processes (Roelofs, 2014; Ueno et al., 2011). 
Ueno et al.'s Lichtheim 2 model proposes the ventral pathway is primarily involved in this function (Ueno et al., 2011) in line with the perception models described above. However, Roelofs' WEAVER++/ARC model proposes a secondary dorsal pathway (D2 section of the Arcuate Fasciculus) connecting the left MTG to the LIFG supports the mapping of lexicalsemantic representations (in the left MTG) onto speech output processes (in the LIFG) (Roelofs, 2014). Our results align better with the predictions of the Lichtheim 2 model, as a crucial role of the dorsal stream (reflected by higher error rates overall in the dorsal than ventral stream) should have been observed in both tasks based on the WEAVER++/ARC's predictions. We note however that these models converge in the idea that the anterior temporal lobe plays an important role in conceptual processing, suggesting that part of the ventral pathway (linking the posterior temporal cortex to the ATL) is critical for lexical-tosemantic mapping across models.

There were some differences observed between tasks that varied depending on the site of testing (cortical vs. subcortical). During ventral stream cortical stimulation, we observed more errors during sentence generation than during PWI, although error rates were very low overall during cortical testing in the PWI. This result may support a role of the ventral stream in semantic-to-lexical mapping, as there were more words to be retrieved overall in sentence generation than PWI. This result could also be taken as an argument in favor of a role of the ventral stream in syntactic processing, needed in sentence generation but not in single word production. A category of models have argued for a role of the ventral stream in supporting this function (Fridriksson et al., 2016; Hickok \& Poeppel, 2004; Saur et al., 2008), and particularly for simple sentences as used here (Friederici et al., 2006, 2012). However, if this were true, similar results should have been observed at the subcortical level. This was not the case in our study as there was no significant effect of Task during subcortical testing in the ventral stream. In addition, all patients with tumors located below the Sylvian fissure made more errors in the PWI than in the sentence generation task. Moreover, converging evidence is brought by the diffusion imaging analyses based on behavioral profiles. The patients who made more errors in the sentence generation task than in the PWI task had dorsal tumor connectivity profiles, while the patients who made more errors in the PWI task than in the sentence generation task showed more diffuse but overall more ventral tumor connectivity. This pattern of results is not in agreement with the theory postulating a role of the ventral stream in syntactic processing for language production and instead supports a role for the dorsal stream in supporting this function (see also supplementary materials for the distribution of grammatical errors in the ventral versus the dorsal stream).

Concerning the subcortical testing results and connectivity maps, we argue that the reason why patients who made more errors in the PWI vs. sentence generation tasks had more ventral connectivity patterns (Figure 5) is due to the presence of the semantic interference manipulation and the necessity to ignore the distractor word in the PWI task. In our study, we did not find an interference effect on error rates, but the PWI task demanded more semantic interference resolution and controlled retrieval than the sentence generation task, in which no such manipulations were present. Previous studies suggest that semantic interference resolution in language production and controlled retrieval and selection of semantic knowledge during word comprehension are supported by ventral stream pathways 
(Harvey \& Schnur, 2015; Harvey, Wei, Ellmore, Hamilton, \& Schnur, 2013). In particular, the inferior frontal occipital fasciculus has been associated with semantic interference resolution in language production (Harvey \& Schnur, 2015), and the uncinate fasciculus has been associated with controlled retrieval and selection of semantic knowledge during word comprehension (Harvey et al., 2013). This is also in line with Roelofs' WEAVER++/ARC model (Roelofs, 2014) according to which the ventral pathway is involved in top-down control aspects of language production. Here, we did not identify the specific pathways supporting these processes but our results are in agreement with the proposal that ventral white matter pathways are recruited by tasks necessitating controlled retrieval and resolution of semantic interference in language production.

\section{Role of the dorsal stream in language production}

Differences between tasks in the dorsal stream only emerged during subcortical testing. There were more errors in the sentence generation task than in the PWI task during subcortical testing and no significant difference between tasks during cortical stimulation for dorsal stream testing (although error rates trended to be lower in PWI than in Sentence Generation there too). In addition, the connectivity maps of the patients who made more errors in the sentence generation task than in the PWI task during subcortical testing were more dorsal.

The sentence generation task involves the production of verbs, whereas the PWI task only involves the production of nouns. A posterior-frontal and parietal versus temporal dissociation has been proposed to underlie verb versus noun processing, respectively (for a review, see Cappa \& Perani, 2003). Thus, verb processing has been proposed to involve more dorsal areas than noun processing. Awake language mapping studies have reported that different cortical sites may be involved in verb and noun processing (Lubrano, Filleron, Démonet, \& Roux, 2014), although not necessarily dissociated along dorsal and ventral streams (Corina et al., 2005). We note that several studies have failed to find anatomical differences between verb and noun processing and report a high degree of overlap between the associated brain activations (for reviews, see Crepaldi et al., 2013; Crepaldi, Berlingeri, Paulesu, \& Luzzatti, 2011).

Hickok (2012) has proposed that the dorsal stream supports sensory-motor feedback and articulatory control in language production. This model proposes that the increased phonemic paraphasias in patients with conduction aphasia is caused by an impaired integration of somatosensory feedback with phonemic planning as a consequence of dorsal stream damage. In our study, the sentence generation task required the production of more words than the PWI task, which required only single word production. Thus, the increased production load may have resulted in an increased recruitment of dorsal pathways in sentence generation, compared to single word production. In support with this proposal, awake language mapping studies have argued for a role of the dorsal stream in phonological encoding (for a review see Duffau, Moritz-Gasser, \& Mandonnet, 2014).

The question in our study is whether the dorsal stream may also support the ordering of words in a sequence, as suggested in language perception by (Bornkessel-Schlesewsky et al., 2015). Indeed, the sentence generation task differs from the PWI task in that the sentence 
generation task requires the subject to order words in a syntactically correct sequence. Syntactic processing is one of the main aspects differentiating dual stream models of language processing (although not addressed in dual-stream language production models, Roelofs, 2014; Ueno et al., 2011). While a category of models argue for a role of the ventral stream in syntactic processing (Fridriksson et al., 2016; Hickok \& Poeppel, 2004; Saur et al., 2008), others argue for a role of the dorsal stream in this function (Bornkessel-Schlesewsky et al., 2015; Wilson et al., 2011). Previous awake language mapping studies addressing syntactic processing are scarce and rarely assessed syntax in the context of language production (for a review see Zanin et al., 2017). However, Leclercq et al. (2010) have shown that the stimulation of the arcuate fasciculus (a dorsal stream pathway) causes syntactic deficits in addition to articulatory deficits, whereas stimulation of the inferior frontal occipital fasciculus (a ventral stream pathway) induces semantic paraphasias. Syntactic errors were also observed following stimulation of other dorsal stream regions: the superior longitudinal fasciculus (Maldonado, Moritz-Gasser, \& Duffau, 2011) and the left inferior parietal lobule (Maldonado, Moritz-Gasser, de Champfleur, et al., 2011). These studies argue for a role of the dorsal stream in syntactic processing in addition to phonological encoding.

A closer look at the types of errors in our study agree with this interpretation as grammatical errors were more frequent during dorsal stream testing (cortical: 18\%; subcortical: 22\%) than during ventral stream testing (cortical: 11\%; subcortical: 6\%) both during cortical stimulation and subcortical resection (see supplementary materials ${ }^{6}$ ). Therefore, our results support dual stream models arguing for a role of the dorsal stream in syntactic processing (Bornkessel-Schlesewsky et al., 2015) and extend these models to language production.

\section{Differences between cortical and subcortical testing}

The division between dorsal and ventral stream function was clearer during subcortical than during cortical testing. Indeed, the Task by Stream interaction during subcortical testing was evident on a patient-by-patient basis: all patients showed more errors the dorsal in the sentence generation task than in the PWI task during dorsal stream testing, and the opposite was true during ventral stream testing. More variable patterns of results were observed at the cortical level. Higher degrees of plasticity have been reported at the cortical level compared to the subcortical level in patients with brain tumors. Indeed, the inter-patient variability has been shown to be high during cortical stimulation in a number of neurosurgical studies (e.g., Corina et al., 2005; Duffau, 2014; Duffau, 2014; Ojemann, 1979; Ojemann, Ojemann, Lettich, \& Berger, 1989; Ojemann, Ojemann, Lettich, \& Berger, 2008; Penfield \& Roberts, 1959; Sanai, Mirzadeh, \& Berger, 2008). At the subcortical level, however, persistent language deficits can be observed depending on the white matter pathway damaged (Caverzasi et al., 2016; Duffau, 2015; Duffau et al., 2002). In addition, preserving white matter pathways in the vicinity of the lesion has been associated with better language outcomes (Caverzasi et al., 2016; Duffau, Gatignol, Mandonnet, Capelle, \& Taillandier, 2008). These studies suggest a greater degree of plasticity at the cortical level compared to the subcortical level, which could explain why our results were generally clearer during

\footnotetext{
${ }^{6}$ We also note that the distributions of phonemic paraphasias or verb versus non-verb errors do not support the other two possible interpretations as strongly, as there were opposite patterns of results at the cortical and subcortical levels for these errors.
} 
subcortical resection than during cortical testing. Indeed, the slow growing tumors (most patients in our study had grade 2 tumors) are likely to have caused substantial reorganization of function at the cortical level. We note that similar observations have been made in studies investigating the neurobiology of language through stroke-induced aphasia and lesionsymptom mapping (e.g., Dronkers, Plaisant, Iba-Zizen, \& Cabanis, 2007; Gaizo et al., 2017; Griffis, Nenert, Allendorfer, \& Szaflarski, 2017). These authors report more severe and longer lasting impairment after disruption of major white matter pathways linking the left temporal lobe to the left frontal lobe compared to isolated cortical damage only (Dronkers et al., 2007; Thiebaut de Schotten et al., 2015). Interestingly, neurosurgical investigations of language have led to the proposal of a hodotopical map of language processing in which the neurobiological basis of language is organized in parallel segregated large-scale corticosubcortical subnetworks underlying different aspects of language processing, and where white matter pathways play a central role in the neurobiology of language (Duffau, MoritzGasser, \& Mandonnet, 2014). In particular, this model associates dorsal stream pathways (in particular the arcuate fasciculus) with phonological encoding, and ventral stream pathways (in particular the inferior frontal occipital fasciculus) with semantic processing. Our results agree with this distinction and additionally suggest dorsal stream pathways support syntactic encoding during sentence generation. Importantly, our study also points to a major and causal role of white matter pathways in language and highlights the need to include these pathways as playing central roles in neurobiological language models.

\section{Limitations of the present study}

One caveat concerning the spatial resolution is worth mentioning. Because of our focus on dissociating the roles of ventral versus dorsal language pathways and due to sparse and spatially-biased spatial sampling inherent to awake language mapping, we collapsed across broad cortical structures for statistical analysis. Therefore, we are unable to make more specific claims regarding the spatial localization of our effects. In addition, only a subset of participants were tested during subcortical resection and stimulation $(\mathrm{n}=10)$, and a subset of those performed both tasks during subcortical testing $(n=7)$. Therefore, our results should be considered as initial steps towards dissociating the roles of the dorsal and ventral streams in language production using the unique approach of awake cortical and subcortical stimulation.

In conclusion, the rare opportunity provided by the assessment of different aspects of language production during awake cortical and subcortical language mapping in patients undergoing tumor resection sheds new light on the organization of language production along ventral and dorsal processing streams. Our results indicate that the ventral and dorsal stream play dissociable roles in language production as in language perception. In particular, in agreement with dual stream language models (Bornkessel-Schlesewsky et al., 2015), our results support that dorsal stream pathways are critical for organizing elements in a sequence, particularly important in the generation of sentences, and that ventral stream pathways are critical for the processing of meaning dependencies, probed here through both the sentence generation task and the picture-word interference task. 


\section{Supplementary Material}

Refer to Web version on PubMed Central for supplementary material.

\section{Acknowledgements}

This research was supported by a post-doctoral grant from the National Institute on Deafness and Other Communication Disorders of the National Institutes of Health under Award Number F32DC013245 to S.K.R., by grants from the Netherlands Organization for Scientific Research under award numbers 446-13-009 and 451-17-003 to V.P., National Defense Science and Engineering Graduate Fellowship awarded to K.M.J., NIH grant 5R01NS066654-05 to R.H., and NINDS grant 2R37NS21135 to R.T.K.. The content is solely the responsibility of the authors and does not necessarily represent the official views of the National Institutes of Health. Finally, we are very thankful to the patients who took part in this study and to their families.

\section{References}

Avants BB, Epstein CL, Grossman M, \& Gee JC (2008). Symmetric diffeomorphic image registration with cross-correlation: evaluating automated labeling of elderly and neurodegenerative brain. Medical Image Analysis, 12(1), 26-41. 10.1016/j.media.2007.06.004 [PubMed: 17659998]

Avants BB, Tustison N, \& Song G. (2009). Advanced normalization tools (ANTS). Insight J, 2, 1-35.

Baayen RH, Davidson DJ, \& Bates DM (2008). Mixed-effects modeling with crossed random effects for subjects and items. Journal of Memory and Language, 59(4), 390-412. 10.1016/j.jml. 2007.12.005

Bates D, Mächler M, Bolker B, \& Walker S. (2014). Fitting Linear Mixed-Effects Models using lme4. arXiv:1406.5823 [Stat]. Retrieved from http://arxiv.org/abs/1406.5823

Berman J, Chung S, Mukherjee P, Hess C, Han E, \& Henry R (2008). Probabilistic streamline q-ball tractography using the residual bootstrap. NeuroImage, 39(1), 215-222. 10.1016/j.neuroimage. 2007.08.021 [PubMed: 17911030]

Bornkessel-Schlesewsky I, Schlesewsky M, Small SL, \& Rauschecker JP (2015). Neurobiological roots of language in primate audition: common computational properties. Trends In Cognitive Sciences, 19(3), 142-150. 10.1016/j.tics.2014.12.008 [PubMed: 25600585]

Brodeur MB, Dionne-Dostie E, Montreuil T, \& Lepage M. (2010). The Bank of Standardized Stimuli (BOSS), a New Set of 480 Normative Photos of Objects to Be Used as Visual Stimuli in Cognitive Research. PLOS ONE, 5(5), e10773 10.1371/journal.pone.0010773 [PubMed: 20532245]

Brysbaert M, \& New B (2009). Moving beyond Kucera and Francis: a critical evaluation of current word frequency norms and the introduction of a new and improved word frequency measure for American English. Behavior Research Methods, 41(4), 977-990. 10.3758/BRM.41.4.977 [PubMed: 19897807]

Bürki A. (2017). Electrophysiological characterization of facilitation and interference in the pictureword interference paradigm. Psychophysiology, 54(9), 1370-1392. 10.1111/psyp.12885 [PubMed: 28470728]

Cappa SF, \& Perani D. (2003). The neural correlates of noun and verb processing. Journal of Neurolinguistics, 16(2), 183-189. 10.1016/S0911-6044(02)00013-1

van Casteren M, \& Davis MH (2006). Mix, a program for pseudorandomization. Behavior Research Methods, 38(4), 584-589. 10.3758/BF03193889 [PubMed: 17393828]

Caverzasi E, Flervey-Jumper SL, Jordan KM, Lobach IV, Li J, Panara V, ... Henry RG (2016). Identifying preoperative language tracts and predicting postoperative functional recovery using HARDI q-ball fiber tractography in patients with gliomas. Journal of Neurosurgery, 125(1), 33-45. 10.3171/2015.6.JNS142203 [PubMed: 26654181]

Chang EF, Raygor KP, \& Berger MS (2015). Contemporary model of language organization: an overview for neurosurgeons. Journal of Neurosurgery, 122(2), 250-261. 10.3171/2014.10.JNS132647 [PubMed: 25423277]

Cohen J, MacWhinney B, Flatt M, \& Provost J. (1993). PsyScope: An interactive graphic system for designing and controlling experiments in the psychology laboratory using Macintosh computers. Behavior Research Methods, Instruments, \& Computers, 25(2), 257-271. 10.3758/BF03204507 
Corina DP, Gibson EK, Martin R, Poliakov A, Brinkley J, \& Ojemann GA (2005). Dissociation of action and object naming: evidence from cortical stimulation mapping. Human Brain Mapping, 24(1), 1-10. 10.1002/hbm.20063 [PubMed: 15593268]

Costa A, Alario FX, \& Caramazza A. (2005). On the categorical nature of the semantic interference effect in the picture-word interference paradigm. Psychonomic Bulletin \& Review, 12(1), 125131. [PubMed: 15948287]

Crepaldi D, Berlingeri M, Cattinelli I, Borghese NA, Luzzatti C, \& Paulesu E. (2013). Clustering the lexicon in the brain: a meta-analysis of the neurofunctional evidence on noun and verb processing. Frontiers In Human Neuroscience, 7, 303 10.3389/fnhum.2013.00303 [PubMed: 23825451]

Crepaldi D, Berlingeri M, Paulesu E, \& Luzzatti C. (2011). A place for nouns and a place for verbs? A critical review of neurocognitive data on grammatical-class effects. Brain and Language, 116(1), 33-49. 10.1016/j.bandl.2010.09.005 [PubMed: 21036389]

Dronkers NF, Plaisant O, Iba-Zizen MT, \& Cabanis EA (2007). Paul Broca's historic cases: high resolution MR imaging of the brains of Leborgne and Lelong. Brain: A Journal of Neurology, 130(Pt 5), 1432-1441. 10.1093/brain/awm042 [PubMed: 17405763]

Duffau H. (2014). Diffuse low-grade gliomas and neuroplasticity. Diagnostic and Interventional Imaging, 95(10), 945-955. 10.1016/j.diii.2014.08.001 [PubMed: 25218490]

Duffau H. (2014). The huge plastic potential of adult brain and the role of connectomics: new insights provided by serial mappings in glioma surgery. Cortex; a Journal Devoted to the Study of the Nervous System and Behavior, 58, 325-337. 10.1016/j.cortex.2013.08.005 [PubMed: 24050218]

Duffau H. (2015). Stimulation mapping of white matter tracts to study brain functional connectivity. Nature Reviews Neurology, 11(5), 255-265. 10.1038/nrneurol.2015.51 [PubMed: 25848923]

Duffau H, Capelle L, Sichez N, Denvil D, Lopes M, Sichez J-P, ... Fohanno D. (2002). Intraoperative mapping of the subcortical language pathways using direct stimulations. An anatomo-functional study. Brain: A Journal of Neurology, 125(Pt 1), 199-214. [PubMed: 11834604]

Duffau H, Gatignol P, Denvil D, Lopes M, \& Capelle L. (2003). The articulatory loop: study of the subcortical connectivity by electrostimulation. Neuroreport, 14(15), 2005-2008. 10.1097/01.wnr. 0000094103.16607.9f [PubMed: 14561939]

Duffau H, Gatignol P, Mandonnet E, Peruzzi P, Tzourio-Mazoyer N, \& Capelle L. (2005). New insights into the anatomo-functional connectivity of the semantic system: a study using corticosubcortical electrostimulations. Brain: A Journal of Neurology, 128(Pt 4), 797-810. 10.1093/brain/ awh423 [PubMed: 15705610]

Duffau H, Moritz-Gasser S, \& Mandonnet E. (2014). A re-examination of neural basis of language processing: proposal of a dynamic hodotopical model from data provided by brain stimulation mapping during picture naming. Brain and Language, 131, 1-10. 10.1016/j.band1.2013.05.011 [PubMed: 23866901]

Duffau H, Peggy Gatignol ST, Mandonnet E, Capelle L, \& Taillandier L. (2008). Intraoperative subcortical stimulation mapping of language pathways in a consecutive series of 115 patients with Grade II glioma in the left dominant hemisphere. Journal of Neurosurgery, 109(3), 461-471. 10.3171/JNS/2008/109/9/0461 [PubMed: 18759577]

Fox J, \& Weisberg S. (2011). An R Companion to Applied Regression (second).

Fridriksson J, den Ouden D-B, Hillis AE, Hickok G, Rorden C, Basilakos A, ... Bonilha L. (2018). Anatomy of aphasia revisited. Brain, 141(3), 848-862. 10.1093/brain/awx363 [PubMed: 29360947]

Fridriksson J, Yourganov G, Bonilha L, Basilakos A, Den Ouden D-B, \& Rorden C. (2016). Revealing the dual streams of speech processing. Proceedings of the National Academy of Sciences of the United States of America, 113(52), 15108-15113. 10.1073/pnas.1614038114 [PubMed: 27956600]

Friederici AD (2012). The cortical language circuit: from auditory perception to sentence comprehension. Trends in Cognitive Sciences, 16(5), 262-268. 10.1016/j.tics.2012.04.001 [PubMed: 22516238]

Friederici AD, Bahlmann J, Fleim S, Schubotz RI, \& Anwander A. (2006). The brain differentiates human and non-human grammars: functional localization and structural connectivity. Proceedings 
of the National Academy of Sciences of the United States of America, 103(7), 2458-2463. 10.1073/pnas.0509389103 [PubMed: 16461904]

Friederici AD, Makuuchi M, \& Bahlmann J. (2009). The role of the posterior superior temporal cortex in sentence comprehension. Neuroreport, 20(6), 563-568. 10.1097/WNR.0b013e3283297dee [PubMed: 19287322]

Gaizo JD, Fridriksson J, Yourganov G, Flillis AE, Hickok G, Misic B, ... Bonilha L. (2017). Mapping Language Networks Using the Structural and Dynamic Brain Connectomes. eNeuro, ENEURO. 0204-17.2017. 10.1523/ENEURO.0204-17.2017

Garyfallidis E, Brett M, Amirbekian B, Rokem A, van der Walt S, Descoteaux M, \& Nimmo-Smith I. (2014). Dipy, a library for the analysis of diffusion MRI data. Frontiers in Neuroinformatics, 8 10.3389/fninf.2014.00008

Glaser WR, \& Düngelhoff FJ (1984). The time course of picture-word interference. Journal of Experimental Psychology. Human Perception and Performance, 10(5), 640-654. [PubMed: 6238124]

Glasser MF, \& Rilling JK (2008). DTI Tractography of the Human Brain's Language Pathways. Cerebral Cortex, 18(11), 2471-2482. 10.1093/cercor/bhn011 [PubMed: 18281301]

Griffis JC, Nenert R, Allendorfer JB, \& Szaflarski JP (2017). Damage to white matter bottlenecks contributes to language impairments after left hemispheric stroke. Neuroimage. Clinical, 14, 552565. 10.1016/j.nicl.2017.02.019 [PubMed: 28337410]

Haller S, Radue EW, Erb M, Grodd W, \& Kircher T. (2005). Overt sentence production in eventrelated fMRI. Neuropsychologia, 43(5), 807-814. 10.1016/j.neuropsychologia.2004.09.007 [PubMed: 15721193]

Harvey DY, \& Schnur TT (2015). Distinct loci of lexical and semantic access deficits in aphasia: Evidence from voxel-based lesion-symptom mapping and diffusion tensor imaging. Cortex; a Journal Devoted to the Study of the Nervous System and Behavior, 67, 37-58. 10.1016/j.cortex. 2015.03.004 [PubMed: 25880795]

Harvey DY, Wei T, Ellmore TM, Hamilton AC, \& Schnur TT (2013). Neuropsychological evidence for the functional role of the uncinate fasciculus in semantic control. Neuropsychoiogia, 51(5), 789801.

Hickok G. (2012). Computational neuroanatomy of speech production. Nature Reviews. Neuroscience, 13(2), 135-145. 10.1038/nrn3158 [PubMed: 22218206]

Hickok G, \& Poeppel D. (2004). Dorsal and ventral streams: a framework for understanding aspects of the functional anatomy of language. Cognition, 92(1-2), 67-99. 10.1016/j.cognition.2003.10.011 [PubMed: 15037127]

Hickok G, \& Poeppel D. (2007). The cortical organization of speech processing. Nature Reviews. Neuroscience, 8(5), 393-402. 10.1038/nrn2113 [PubMed: 17431404]

Indefrey P, Brown CM, Hellwig F, Amunts K, Herzog H, Seitz RJ, \& Hagoort P. (2001). A neural correlate of syntactic encoding during speech production. Proceedings of the National Academy of Sciences of the United States of America, 98(10), 5933-5936. 10.1073/pnas.101118098 [PubMed: 11331773]

Jaeger TF (2008). Categorical data analysis: Away from ANOVAs (transformation or not) and towards logit mixed models. Journal of Memory and Language, 59(4), 434-446. 10.1016/j.jml.2007.11.007 [PubMed: 19884961]

Jenkinson M, Beckmann CF, Behrens TEJ, Woolrich MW, \& Smith SM (2012). FSL. Neuroimage, 62(2), 782-790. 10.1016/j.neuroimage.2011.09.015 [PubMed: 21979382]

Jordan KM, Keshavan A, Caverzasi E, Osorio J, Papinutto N, Amirbekian B, ... Henry RG (2017). Investigating The Functional Consequence Of White Matter Damage: An Automatic Pipeline To Create Longitudinal Disconnection Tractograms. bioRxiv, 140137 10.1101/140137

Leclercq D, Duffau H, Delmaire C, Capelle L, Gatignol P, Ducros M, ... Lehéricy S (2010). Comparison of diffusion tensor imaging tractography of language tracts and intraoperative subcortical stimulations. Journal of Neurosurgery, 112(3), 503-511. 10.3171/2009.8.JNS09558 [PubMed: 19747052] 
Leemans A, \& Jones DK (2009). The B-matrix must be rotated when correcting for subject motion in DTI data. Magnetic Resonance in Medicine, 61(6), 1336-1349. 10.1002/mrm.21890 [PubMed: 19319973]

Lichteim L. (1885). On Aphasial. Brain, 7(4), 433-484. 10.1093/brain/7.4.433

Lubrano V, Filleron T, Demonet J-F, \& Roux F-E (2014). Anatomical correlates for category-specific naming of objects and actions: a brain stimulation mapping study. Human Brain Mapping, 35(2), 429-443. 10.1002/hbm.22189 [PubMed: 23015527]

Lupker SJ (1979). The semantic nature of response competition in the picture-word interference task. Memory \& Cognition, 7(6), 485-495. 10.3758/BF03198265

Maldonado IL, Moritz-Gasser S, de Champfleur NM, Bertram L, Moulinié G, \& Duffau H (2011). Surgery for gliomas involving the left inferior parietal lobule: new insights into the functional anatomy provided by stimulation mapping in awake patients. Journal of Neurosurgery, 115(4), 770-779. 10.3171/2011.5.JNS112 [PubMed: 21699481]

Maldonado IL, Moritz-Gasser S, \& Duffau H. (2011). Does the left superior longitudinal fascicle subserve language semantics? A brain electrostimulation study. Brain Structure \& Function, 216(3), 263-274. 10.1007/s00429-011-0309-x [PubMed: 21538022]

Mandonnet E, Nouet A, Gatignol P, Capelle L, \& Duffau H. (2007). Does the left inferior longitudinal fasciculus play a role in language? A brain stimulation study. Brain: A Journal of Neurology, 130(Pt 3), 623-629. 10.1093/brain/awl361 [PubMed: 17264096]

Martino J, Brogna C, Robles SG, Vergani F, \& Duffau H (2010). Anatomic dissection of the inferior fronto-occipital fasciculus revisited in the lights of brain stimulation data. Cortex, 46(5), 691-699. 10.1016/j.cortex.2009.07.015 [PubMed: 19775684]

Masterson J, \& Druks J. (1998). Description of a set of 164 nounsand 102 verbs matched for printed word frequency, familiarityand age-of-acquisition. Journal of Neurolinguistics, 11(A), 331-354. 10.1016/S0911-6044(98)00023-2

Ojemann GA (1979). Individual variability in cortical localization of language. Journal of Neurosurgery, 50(2), 164-169. 10.3171/jns.1979.50.2.0164 [PubMed: 430127]

Ojemann G, Ojemann J, Lettich E, \& Berger M. (1989). Cortical language localization in left, dominant hemisphere. An electrical stimulation mapping investigation in 117 patients. Journal of Neurosurgery, 71(3), 316-326. 10.3171/jns.1989.71.3.0316 [PubMed: 2769383]

Ojemann G, Ojemann J, Lettich E, \& Berger M. (2008). Cortical language localization in left, dominant hemisphere. An electrical stimulation mapping investigation in 117 patients. 1989. Journal of Neurosurgery, 108(2), 411-421. 10.3171/JNS/2008/108/2/0411 [PubMed: 18240946]

Penfield W, \& Roberts L. (1959). Speech and Brain-mechanisms. Princeton University Press.

Piai V, \& Knight RT (2017). Lexical selection with competing distractors: Evidence from left temporal lobe lesions. Psychonomic Bulletin \& Review, 10.3758/s13423-017-1301-0

Piai V, Roelofs A, Acheson DJ, \& Takashima A. (2013). Attention for speaking: domain-general control from the anterior cingulate cortex in spoken word production. Frontiers in Human Neuroscience, 7, 832 10.3389/fnhum.2013.00832 [PubMed: 24368899]

Piai V, Roelofs A, Jensen O, Schoffelen J-M, \& Bonnefond M. (2014). Distinct patterns of brain activity characterise lexical activation and competition in spoken word production. PloS One, 9(2), e88674 10.1371/journal.pone.0088674 [PubMed: 24558410]

Piai V, Roelofs A, \& Schriefers H. (2015). Task choice and semantic interference in picture naming. Acta Psychologica, 157(Supplement C), 13-22. 10.1016/j.actpsy.2015.02.003 [PubMed: 25703606]

Pinheiro JC, \& Bates DM (n.d.). Mixed-effects models in S and S-PLUS (2000th ed.). Springer Science and Business Media.

Pylkkänen L, Bemis DK, \& Blanco Elorrieta E. (2014). Building phrases in language production: An MEG study of simple composition. Cognition, 133(2), 371-384. 10.1016/j.cognition.2014.07.001 [PubMed: 25128795]

Rauschecker JP, \& Scott SK (2009). Maps and streams in the auditory cortex: nonhuman primates illuminate human speech processing. Nature Neuroscience, 12(6), nn.2331 10.1038/nn.2331 
Rauschecker JP, \& Tian B. (2000). Mechanisms and streams for processing of "what" and "where" in auditory cortex. Proceedings of the National Academy of Sciences of the United States of America, 97(22), 11800-11806. 10.1073/pnas.97.22.11800 [PubMed: 11050212]

Roelofs A. (2014). A dorsal-pathway account of aphasic language production: the WEAVER++/ARC model. Cortex; a Journal Devoted to the Study of the Nervous System and Behavior, 59, 33-48. 10.1016/j.cortex.2014.07.001 [PubMed: 25128898]

Roelofs A, \& Piai V. (2015). Aspects of competition in word production: reply to Mahon and Navarrete. Cortex; a Journal Devoted to the Study of the Nervous System and Behavior, 64, 420424. 10.1016/j.cortex.2014.10.016 [PubMed: 25726474]

Roelofs A, \& Piai V. (2017). Distributional analysis of semantic interference in picture naming. Quarterly Journal of Experimental Psychology (2006), 70(4), 782-792. 10.1080/17470218.2016.1165264 [PubMed: 26967984]

Wang Ruopeng, Wedeen Van J.. (n.d.). TrackVis.org. Martinos Center for Biomedical Imaging, Massachusetts General Hospital.

Sanai N, Mirzadeh Z, \& Berger MS (2008). Functional outcome after language mapping for glioma resection. The New England Journal of Medicine, 358(1), 18-27. 10.1056/NEJMoa067819 [PubMed: 18172171]

Saur D, Kreher BW, Schnell S, Kümmerer D, Kellmeyer P, Vry M-S, ... Weiller C. (2008). Ventral and dorsal pathways for language. Proceedings of the National Academy of Sciences of the United States of America, 105(46), 18035-18040. 10.1073/pnas.0805234105 [PubMed: 19004769]

Segaert K, Menenti L, Weber K, Petersson KM, \& Hagoort P. (2012). Shared Syntax in Language Production and Language Comprehension-An fMRI Study. Cerebral Cortex, 22(7), 1662-1670. 10.1093/cercor/bhr249 [PubMed: 21934094]

Smith SM (2002). Fast robust automated brain extraction. Human Brain Mapping, 17(3), 143-155. 10.1002/hbm.10062 [PubMed: 12391568]

Snodgrass JG, \& Vanderwart M. (1980). A standardized set of 260 pictures: Norms for name agreement, image agreement, familiarity, and visual complexity. Journal of Experimental Psychology: Human Learning and Memory, 6(2), 174-215. 10.1037/0278-7393.6.2.174 [PubMed: 7373248]

Thiebaut de Schotten M, Dell'Acqua F, Ratiu P, Leslie A, Howells H, Cabanis E, ... Catani M. (2015). From Phineas Gage and Monsieur Leborgne to H.M.: Revisiting Disconnection Syndromes. Cerebral Cortex (New York, N.Y.: 1991), 25(12), 4812-4827. 10.1093/cercor/bhv173

Tippett DC, \& Hillis AE (2016). Chapter 73 - Vascular Aphasia Syndromes In Hickok G \& Small SL (Eds.), Neurobiology of Language (pp. 913-922). San Diego: Academic Press Retrieved from http://www.sciencedirect.com/science/article/pii/B9780124077942000730

Ueno T, Saito S, Rogers TT, \& Lambon Ralph MA (2011). Lichtheim 2: synthesizing aphasia and the neural basis of language in a neurocomputational model of the dual dorsal-ventral language pathways. Neuron, 72(2), 385-396. 10.1016/j.neuron.2011.09.013 [PubMed: 22017995]

Ungerleider L, \& Mishkin M (1982). Two cortical visual systems In Analysis of Visual Behavior. Cambridge, Mass: MIT Press.

Wilson SM, Galantucci S, Tartaglia MC, Rising K, Patterson DK, Henry ML, ... Gorno-Tempini ML (2011). Syntactic processing depends on dorsal language tracts. Neuron, 72(2), 397-403. 10.1016/ j.neuron.2011.09.014 [PubMed: 22017996]

Zanin E, Riva M, Bambini V, Cappa SF, Magrassi L, \& Moro A. (2017). The contribution of surgical brain mapping to the understanding of the anatomo-functional basis of syntax: A critical review. Neurological Sciences, 38(9), 1579-1589. 10.1007/s10072-017-3016-4 [PubMed: 28624915]

de Zubicaray GI, Wilson SJ, McMahon KL, \& Muthiah S. (2001). The semantic interference effect in the picture-word paradigm: An event-related fMRI study employing overt responses. Human Brain Mapping, 14(A), 218-227. 10.1002/hbm.1054 [PubMed: 11668653] 


\section{Highlights:}

- $\quad$ The dorsal stream may support sentence formation in language processing.

- Direct cortical and subcortical stimulation was used during awake language mapping.

- Subcortically, patients made more errors in sentence vs. single word production.

- This was true in the dorsal stream only, supporting a role in sentence formation.

- Connectivity maps also support a dorsal/ventral division in language production. 


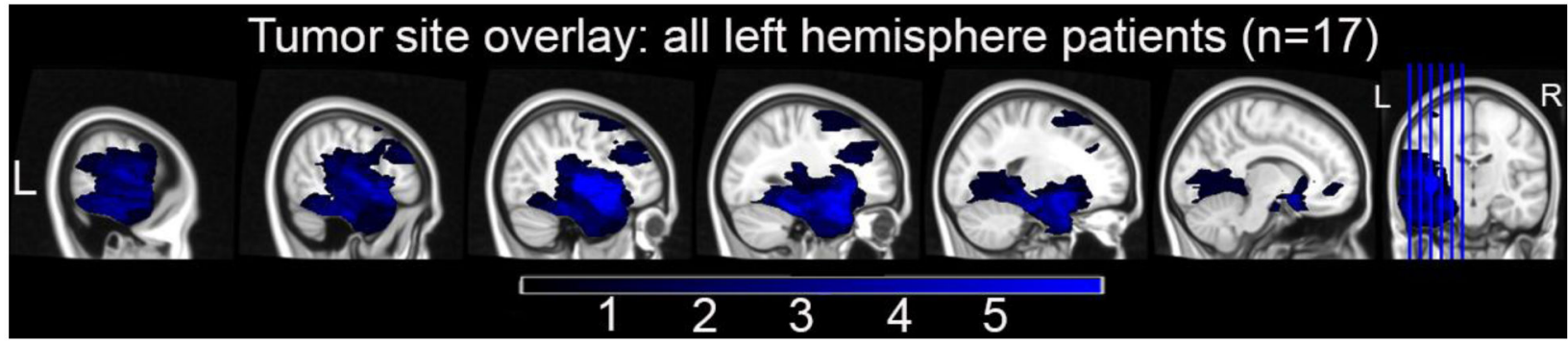

Figure 1:

Overlay of tumor sites in standard MNI space of the 17 left hemisphere patients after reconstruction. The color coding indicates the amount of overlap between the different patients' lesions (from light blue for the highest overlap and dark blue for the lowest overlap). $\mathrm{L}=$ left, $\mathrm{R}=$ right. 


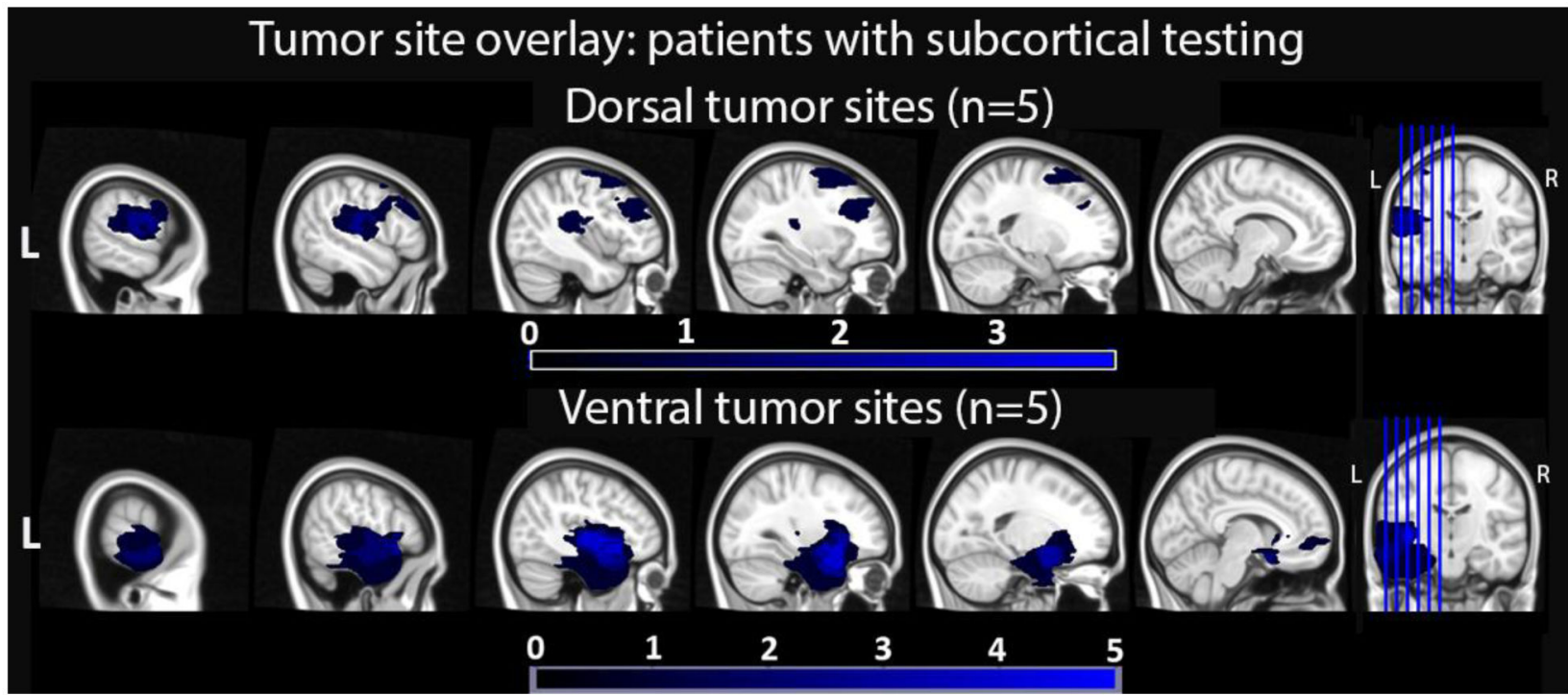

Figure 2:

Overlay of tumor sites in standard MNI space of the 10 patients who underwent testing during subcortical resection by group (top: overlay for the 5 patients with tumors above the Sylvian fissure; bottom: overlay for the 5 patients with tumors below the Sylvian fissure). The color coding indicates the amount of overlap between the different patients' lesions (from light blue for the highest overlap and dark blue for the lowest overlap). L = left. 


\section{Cortical stimulation results per patient} Dorsal stream testing

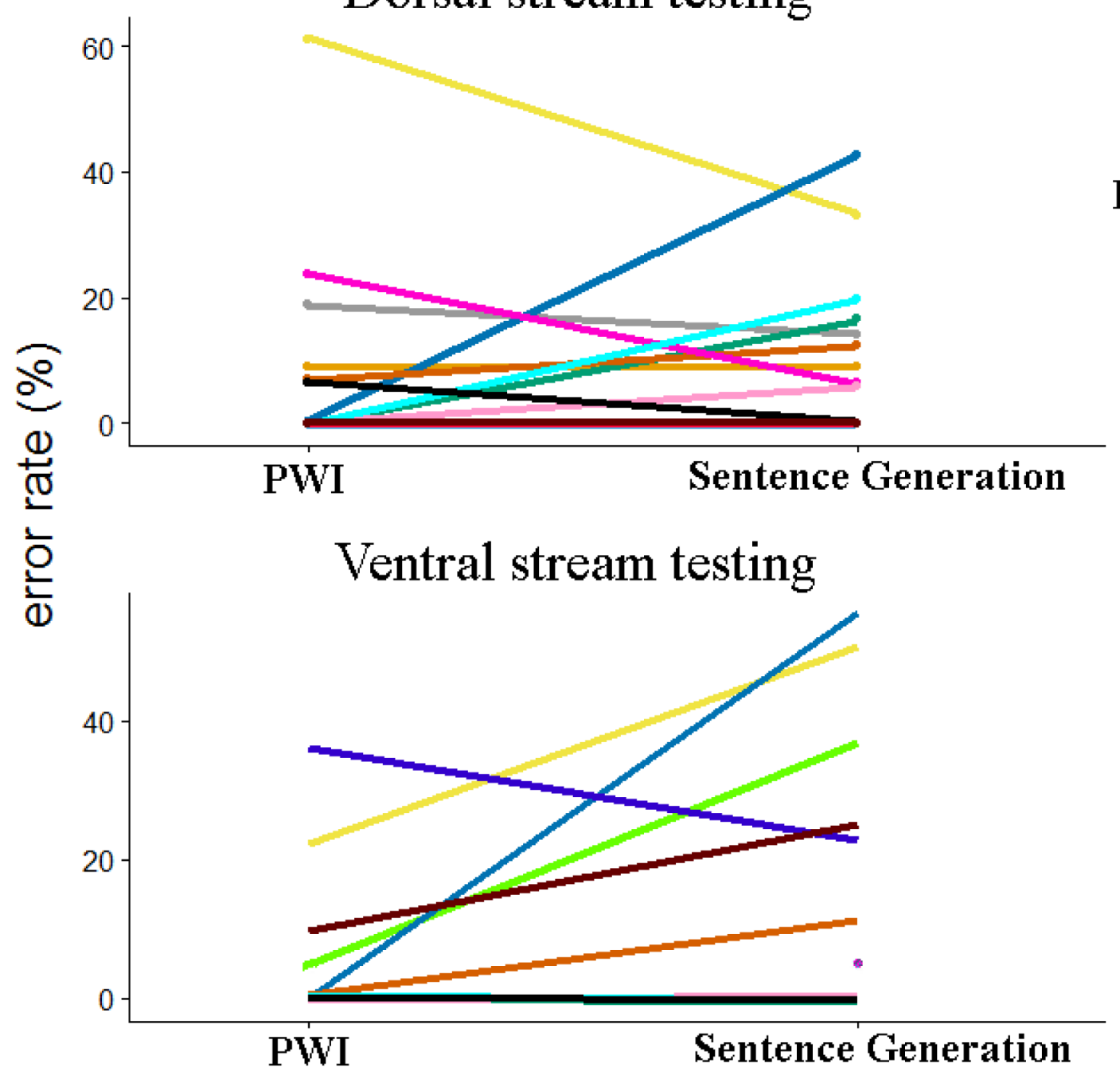

Patients

Figure 3:

Behavioral results during cortical stimulation per patient, task, and stream (top: dorsal stream; bottom: ventral stream). 


\section{Subcortical testing results per patient Dorsal stream testing}

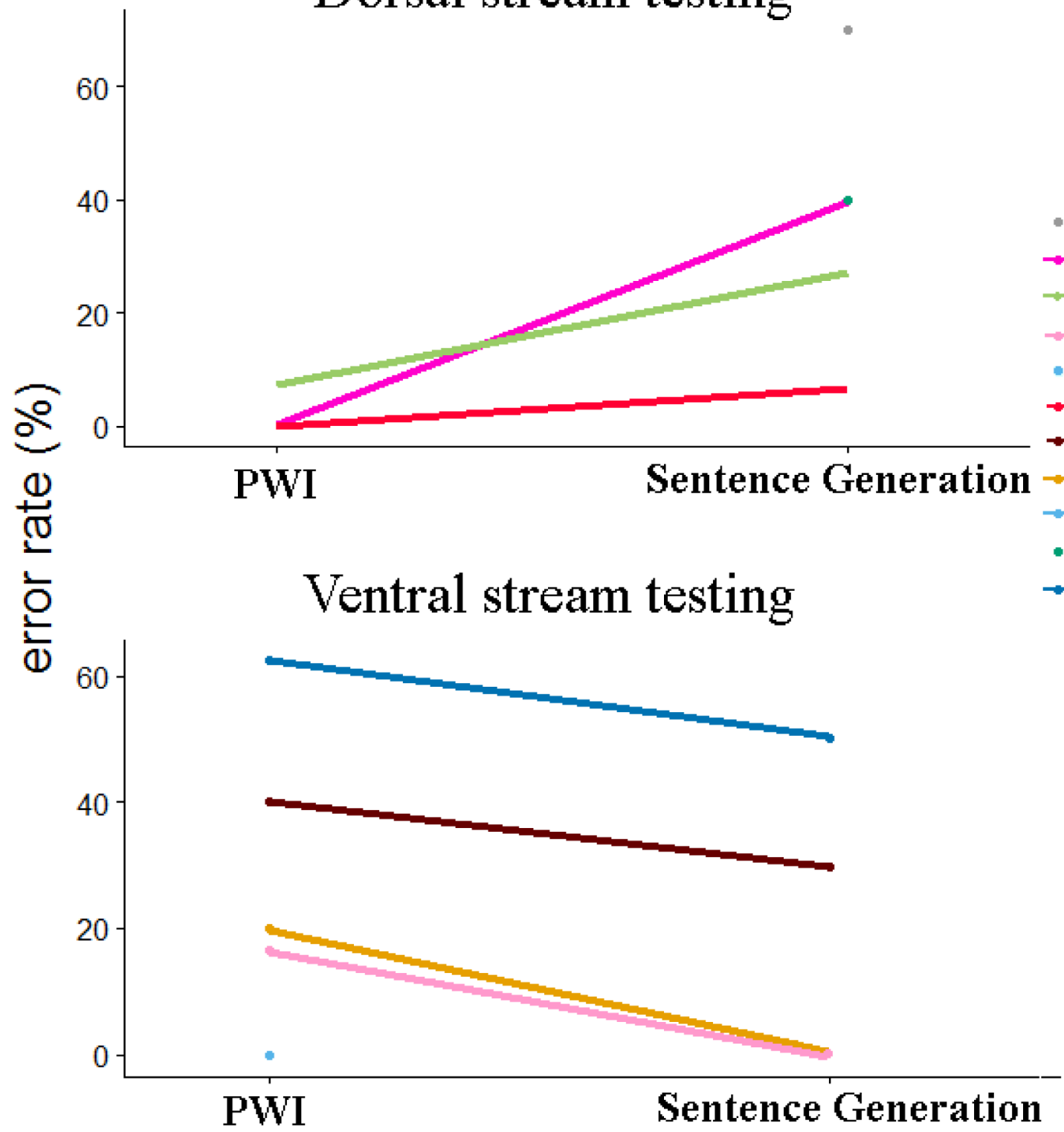

Figure 4:

Behavioral results during subcortical stimulation per patient, task, and stream (top: dorsal stream; bottom: ventral stream). The results for patients who were only tested in one experiment are represented by dots (Pt1, Pt15, Pt6). 


\section{Connectivity maps associated with tumor location per group based on behavioral pattern}

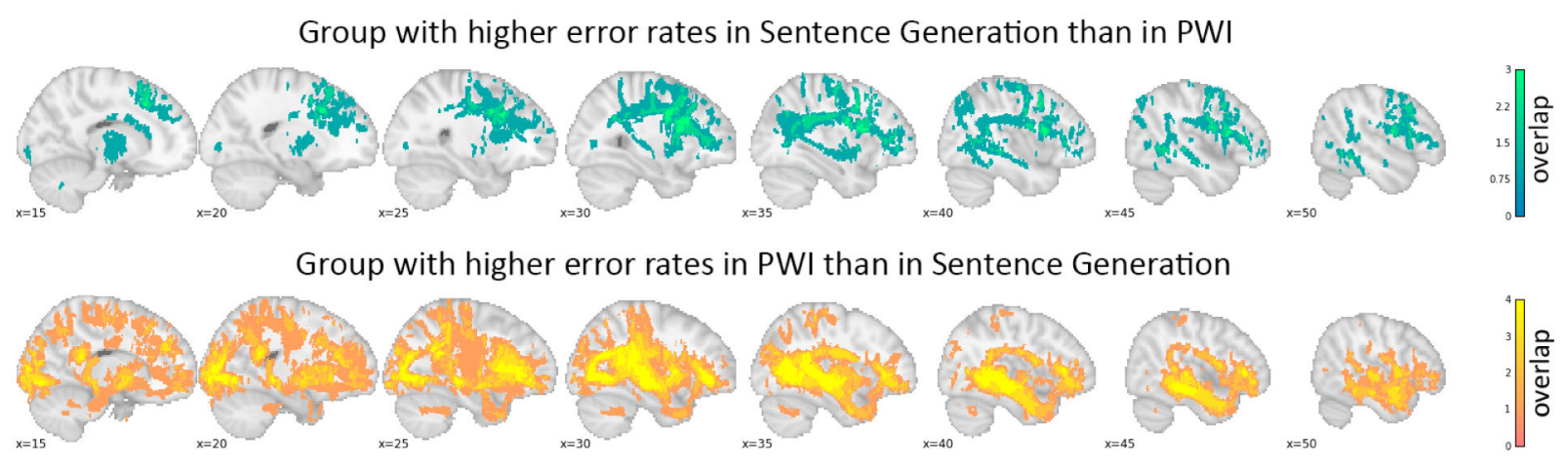

Figure 5:

Connectivity maps in MNI space associated with tumor locations for the 3 patients with higher error rates in Sentence Generation than in PWI during subcortical testing (top), and for the 4 patients with higher error rates in PWI than Sentence Generation during subcortical testing (bottom). The tumor reconstruction volume was targeted from a whole-brain streamline dataset; voxels connected to the tumor volume were mapped here as described in 2.5. Lighter colors indicate higher overlap between patients. 
Table 1:

Etiology, tumor grade, and resected tumor location for the 17 patients included in the study.

\begin{tabular}{|l|l|l|l|}
\hline Patient \# & Pathology & Grade & Resected tumor location \\
1 & Oligodendroglioma & 2 & left inferior parietal lobe \\
2 & Meningioangiomatosis & NA & left middle frontal lobe \\
4 & Oligoastrocytoma & 2 & left inferior frontal lobe \\
5 & Anaplastic astrocytoma & 3 & left inferior frontal lobe and anterior insula \\
6 & Glioblastoma & 4 & left inferior and middle temporal gyri \\
7 & Oligodendroglioma & 2 & left inferior frontal and anterior temporal lobes \\
8 & Oligodendroglioma & 2 & left inferior frontal lobe \\
9 & Anaplastic astrocytoma & 3 & left temporal lobe \\
10 & Oligodendroglioma & 2 & left insula, medial temporal lobe, and basal forebrain \\
11 & Diffuse astrocytoma & 2 & left insular centered, anterior temporal lobe and posterior IFG involved. \\
12 & Diffuse astrocytoma & 2 & left posterior hippocampus \\
13 & Ganglioglioma & 1 & left mid-hippocampus \\
15 & Oligodendroglioma & 2 & left inferior posterior frontal lobe \\
16 & Glioblastoma & 4 & left mid- and superior temporal lobe \\
17 & Oligodendroglioma & 2 & left inferior frontal lobe \\
18 & Oligoastrocytoma & 2 & left insula, anterior temporal, inferior frontal lobes \\
19 & Astrocytoma & 2 & left inferior and middle temporal gyri, parahippocampal gyrus \\
\hline
\end{tabular}


Table 2:

Classification of cortical stimulation sites along the dorsal or ventral language processing streams

\begin{tabular}{|l|}
\hline Dorsal regions \\
- Posterior superior temporal gyrus (pSTG) \\
- All parietal regions \\
- Miderior frontal gyrus pars opercularis (IFGop) \\
- Primary motor gyrus (MFG)
\end{tabular}

\section{Ventral regions}

- Middle superior temporal gyrus (mSTG)

- Anterior superior temporal gyrus (aSTG)

- Middle temporal gyrus (MTG)

- Inferior temporal gyrus (ITG)

- Inferior frontal gyrus pars orbitalis (IFGorb)

- Inferior frontal gyrus pars triangularis (IFGtri) 
Table 3:

Median error rate and inter-quartile $\left(1^{\text {st }}-3^{\text {rd }}\right)$ interval (IQI) per Task and Stream for testing performed during cortical stimulation.

\begin{tabular}{cccc}
\hline & $\begin{array}{c}\text { Dorsal stream } \\
\text { cortical regions }\end{array}$ & $\begin{array}{c}\text { Ventral stream } \\
\text { cortical regions }\end{array}$ & Median \\
Sentence Generation & $5.88 \%$ & $9.09 \%$ & $6.27 \%$ \\
Picture-word Interference & $\mathrm{IQI}=[0 \%-16.67 \%]$ & $\mathrm{IQI}=[0 \%-25.00 \%]$ & $\mathrm{IQI}=[0 \%-19.17 \%]$ \\
& $\mathrm{IQI}=[0 \%-19.17 \%]$ & $\mathrm{IQI}=[0 \%-6.00 \%]$ & $\mathrm{IQI}=[0 \%-8.10 \%]$ \\
Median & $0 \%$ & $0 \%$ & \\
& $\mathrm{IQI}=[0 \%-16.67 \%]$ & $\mathrm{IQI}=[0 \%-18.25 \%]$ & \\
\hline
\end{tabular}

Brain Lang. Author manuscript; available in PMC 2020 April 01. 
Table 4:

Median error rates and inter-quartile ranges per Task and Stream for testing performed during subcortical resection.

\begin{tabular}{cccc}
\hline & Dorsal pathways territory & Ventral pathways territory & Overall Median \\
Sentence & $40 \%$ & $14.81 \%$ & $29.63 \%$ \\
Generation & $\mathrm{IQI}=[27.27 \%-40.00 \%]$ & $\mathrm{IQI}=[0.00 \%-34.72 \%]$ & $\mathrm{IQI}=[6.45 \%-40.00 \%]$ \\
$\begin{array}{c}\text { Picture-word } \\
\text { Interference }\end{array}$ & $\mathrm{IQI}=[0.00 \%-3.57 \%]$ & $\mathrm{IQI}=[16.67 \%-40.00 \%]$ & $\mathrm{IQI}=[0.00 \%-25.00 \%]$ \\
Overall Median & $17.21 \%$ & $20.00 \%$ & \\
& $\mathrm{IQI}=[4.84 \%-40.00 \%]$ & $\mathrm{IQI}=[0.00 \%-40.00 \%]$ & \\
\hline
\end{tabular}

